\section{Avaliação de um programa de controle da asma em unidades de saúde da família}

\author{
Evaluation of an asthma control program in family \\ health units
}

\footnotetext{
1 Diretoria de Ações em Saúde, Autarquia Municipal de Saúde, Londrina, Brasil. 2 Programa de Pós-graduação em Saúde Coletiva, Universidade Estadual de Londrina, Londrina, Brasil. ${ }^{3}$ Departamento de Clínica Médica, Universidade Estadual de Londrina, Londrina, Brasil.

Correspondência T. A. Carmo

Diretoria de Ações em Saúde, Autarquia Municipal de Saúde de Londrina. Rua Santa Catarina 584, Londrina, $P R$ 86010-470, Brasil. tatianecarmo@yahoo.com.br
}

\begin{abstract}
The objective of this study was to evaluate the asthma control program in Londrina, Paraná State, Brazil. This is a cross-sectional study, comparing a family health unit (USF) with a consolidated program with two USFs without one. Interviews were performed with 313 asthma patients, 168 from the USF with a consolidated program and 145 from the USFs without one. In the studied sample, there were significant differences $(p<0.001)$ in the use of bronchodilators and in the number of emergency department visits between the USF with and without a consolidated program. Among the patients enrolled in the USF with a consolidated program, 55.4\% reported the use of bronchodilator, in comparison with $74.5 \%$ of those enrolled in the two USF without a consolidated program. Respectively $29.2 \%$ and $55.9 \%$ of the patients from the USFs with and without a consolidated program needed emergency department care. A well-organized asthma control program may result in reduction of emergency department visits due to asthma exacerbation, thus contributing to improving health indicators and quality of life.
\end{abstract}

Asthma; Program Evaluation; Emergency Medical Services; Hospitalization
Tatiane Almeida do Carmo 1

Selma Maffei de Andrade 2

Alcindo Cerci Neto 1,3

\section{Introdução}

A asma é uma doença crônica que apresenta prevalência elevada e crescente, com importante impacto na demanda dos serviços de saúde. Um estudo multicêntrico, o International Study for Asthma and Allergies in Childhood (ISSAC), realizado na América Latina e abrangendo a faixa etária entre 13 e 14 anos, encontrou prevalência de $16 \%$ no Brasil 1. Em outra investigação, realizada com crianças e adolescentes em várias cidades brasileiras, as prevalências variaram de $6 \%$ a $27,1 \%$, sendo a menor prevalência observada em Campos Gerais (Minas Gerais) e a mais elevada, em Salvador (Bahia) 2. Na cidade de Londrina, Estado do Paraná, a prevalência encontrada de asma entre escolares foi de $22 \% 3$.

Segundo dados do Ministério da Saúde, a asma é responsável por cerca de 350 mil internações e mais de 2 mil óbitos anualmente, sendo responsável também por $5 \%$ a $10 \%$ das mortes por causas respiratórias, além de grande demanda de difícil dimensionamento por assistência ambulatorial e de urgência 4 . A asma ocupa o terceiro lugar entre as causas de internação hospitalar 5. Apesar dos avanços no conhecimento sobre a doença, ela ainda é subdiagnosticada, muitas vezes baseada no atendimento de emergência 6 .

A asma pode ser controlada por uma série de medidas. Segundo o GINA (Global Initiative for Asthma) 7, documento elaborado por autoridades internacionais, o controle da asma pode 
ser realizado por medidas de controle ambiental, por ações de educação para pacientes e seus familiares e pelo uso de medicações, o que pode ser mais facilmente realizado com a implementação de programas de controle de asma.

Oliveira et al. 8 e Mendez et al. 9 demonstraram que a implementação de programas de educação dirigidos a portadores de asma leva à redução das crises asmáticas, diminuindo o número de hospitalizações e de visitas ao pronto-socorro, além de proporcionar melhoria da qualidade de vida desses pacientes.

A asma é considerada um problema de saúde pública, o que tem estimulado a realização de estudos e a implantação de programas de controle, que têm surgido por todo o país ${ }^{10}$. No Brasil, existem algumas iniciativas em programas de controle da asma. Mesmo assim, ainda não existe um panorama ou um indicador que demonstre a real efetividade dessas ações na população que se quer beneficiar.

Em Londrina, em 2002, foi implantado um programa de controle da asma (programa "Respira Londrina”), com o objetivo de prevenir a ocorrência de crises asmáticas e estabelecer estratégias para atender pacientes portadores de asma na atenção primária, pois, até então, toda a demanda era direcionada a especialistas e a atenção se concentrava nas pessoas em situações de crise ${ }^{10}$. O projeto teve início em uma unidade de saúde da família (piloto), com treinamento da equipe, busca ativa de "chiadores" na comunidade por agentes comunitários de saúde e classificação clínica da gravidade da asma, para posterior acompanhamento multiprofissional. Posteriormente, houve expansão do programa, com implantação dele, de forma semelhante, em todas as 52 unidades de saúde da família (USF) do município até o final do ano de 2004.

Nas USF, as ações do programa "Respira Londrina” recomendam atenção multiprofissional aos portadores de asma de todas as idades, busca ativa de pacientes, acesso à medicação necessária, formação de grupos de pacientes e/ou familiares para ações educativas multiprofissionais e visitas domiciliares para orientações sobre controle ambiental e uso dos dispositivos inalatórios. A atenção secundária aos casos de asma grave ou de difícil controle é proporcionada pelo próprio serviço municipal de saúde, em uma policlínica de especialidades que conta com pneumologista e pneumopediatra. Há, ainda, um Conselho Consultivo em Asma (CCA), responsável pelos treinamentos das equipes e elaboração de material técnico, que acompanha a situação do programa em cada USF, por meio de indicadores de cobertura (número de inscritos esperados em comparação à prevalência estimada de asma), de consumo de medicamentos e de existência de atividades programáticas, como formação de grupos de pacientes e/ou familiares e realização de visitas domiciliares específicas para atenção às pessoas com asma 10.

Muitos municípios paranaenses e até mesmo do país ainda não possuem programas de controle da asma. A avaliação do impacto de um programa de controle da asma é, desse modo, de fundamental importância, já que pode demonstrar aos gestores as vantagens de sua implantação e até mesmo reforçar a manutenção de programas de controle deste agravo em municípios que já o implantaram. Este estudo tem como objetivo avaliar resultados/impacto do programa de controle da asma de Londrina, em Unidades de Saúde da Família.

\section{Métodos}

Foi realizado estudo transversal, no período compreendido entre meados de fevereiro e início de maio de 2007, com portadores de asma inscritos no programa "Respira Londrina” em USF do Município de Londrina.

Cabe ressaltar que o objetivo inicial do estudo era o de avaliar o impacto do programa comparando os resultados em USF com e sem o programa implantado. Todavia, à época da realização do presente estudo, todas as USF contavam com o programa implantado, embora em fases diferentes de consolidação, conforme avaliação do CCA do programa. Tal avaliação é feita para cada USF com base nos seguintes parâmetros: proporção de inscritos no Programa em relação ao número esperado, consumo de medicamentos e existência de atividades programáticas voltadas à população asmática. $\mathrm{O}$ número esperado de inscritos, segundo profissionais do CCA, considera uma prevalência geral de asma de 10\% (Organização Pan-Americana da Saúde. Doenças respiratórias crônicas. http://new.paho. org/bra/index2.php?option=com_content\&do_ pdf $=1 \& i d=581$, acessado em 10/Ago/2010), com $50 \%$ de busca aos serviços de saúde (casos de asma não intermitente) e, desses, $70 \%$ de usuários de serviços do Sistema Único de Saúde (SUS). O processo de avaliação das USF compreendia visitas locais a essas unidades, com entrevistas com os profissionais de saúde, além de análise dos relatórios produzidos. A informação sobre a fase de consolidação do programa de cada USF foi obtida no CCA.

Considerando essa avaliação, foram selecionadas intencionalmente três USF cujos programas estavam em fases diferentes de consolidação, contemplando uma avaliada como progra- 
ma consolidado e outras duas como programa não consolidado. A necessidade de incluir duas USF com programa não consolidado deveu-se ao número pequeno de pacientes inscritos nessas unidades. A proporção de inscritos em relação ao número esperado na USF considerada com programa consolidado foi de $55 \%$, enquanto nas USF com programa não consolidado de apenas $19 \%$.

A USF considerada com programa consolidado selecionada está localizada na região norte do município, possui uma população aproximada de 19 mil habitantes e foi a primeira USF a ter implantado o programa de controle da asma do município (USF piloto) 11. Para o cálculo do tamanho da amostra desta USF (total de 358 pacientes), baseou-se em uma prevalência de $50 \%$, a qual possibilita maior tamanho amostral possível, e erro de 5\%, acrescentando-se $15 \%$ para eventuais perdas e recusas. Assim, foram selecionados 212 indivíduos a serem entrevistados. Para a seleção dos entrevistados, foi realizada amostragem aleatória casual simples com base nas fichas de cadastro da USF.

Como já mencionado, em razão do menor número de pacientes cadastrados, houve necessidade de se selecionar duas unidades para compor o número aproximado da amostra de pacientes da USF com programa consolidado. As USF consideradas como programa não consolidado estão localizadas na região oeste do município e possuem populações de aproximadamente 15 mil e 10 mil habitantes 11 . Todos os inscritos no programa de ambas as USF, após aplicados os critérios de exclusão, foram selecionados, sendo tratados em conjunto como cadastrados em USF com programa não consolidado (165 pacientes).

Foram considerados critérios gerais de exclusão: óbitos, mudanças de endereços para áreas de outras USF, outros diagnósticos que não fossem o de asma e crianças menores de dois anos (bebê chiador). Além disso, na USF com programa consolidado, adotou-se como critério de exclusão o tempo inferior a quatro meses de cadastramento no programa por conta da necessidade de se observar um tempo mínimo para avaliação do impacto do programa. Em uma das USF com programa não consolidado, foram ainda excluídos os asmáticos que participaram do teste piloto e os que residiam em uma microárea em que o nível de violência não permitia a entrada dos pesquisadores de campo.

A coleta de dados foi realizada pela autora principal deste estudo e por uma equipe de cinco acadêmicos dos cursos de medicina e enfermagem previamente treinados e supervisionados. Foi realizada checagem por telefone, pela autora principal, de $10 \%$ das entrevistas.
Para análise, as variáveis foram agrupadas tendo como referência a proposta de Donabedian 12 sobre avaliação de qualidade de atenção à saúde, composta por indicadores de estrutura, processo e resultados. Estrutura refere-se aos atributos relativamente estáveis dos serviços, tais como equipamentos, recursos materiais, financeiros e humanos. Processo denota o que realmente é feito no processo de atenção, englobando a forma como pacientes procuram e usam o serviço e as atividades desenvolvidas pelos profissionais de saúde. Resultados, nessa perspectiva, referem-se aos efeitos da atenção na saúde de pacientes e populações 12 .

Considerando o objetivo principal do presente estudo de avaliar os resultados do programa de controle da asma de Londrina, os indicadores de resultado/impacto foram os seguintes: ocorrência de internações hospitalares por doenças respiratórias nos últimos 12 meses, atendimentos de urgência nos últimos noventa dias e condição no programa à época da pesquisa (tratamento, alta e abandono). Abandono do programa foi definido como o não-comparecimento ao serviço de saúde após trinta dias da data agendada para retorno entre pacientes que necessitavam usar medicação ou para consulta médica; a condição alta do programa foi assim definida para os casos que receberam alta médica, que, conforme protocolo, é dada a pacientes com a asma sob controle, que não necessitavam usar medicação e que já haviam participado de todas as atividades educativas propostas pelo programa. Pacientes que ainda estavam inscritos e com retorno agendado sem atraso de trinta dias ou mais foram considerados como em tratamento.

As variáveis relativas ao processo de atenção foram: existência de grupos para asmáticos, atendimento fisioterápico, realização de visita domiciliar para orientações de controle ambiental, uso de corticosteróides inalatórios nos últimos trinta dias e uso de broncodilatador nos últimos noventa dias. Esses prazos, assim como os dos indicadores de resultados, foram definidos para reduzir vieses de memória, proporcionando mais tempo de recordatório aos eventos mais marcantes.

Outras variáveis analisadas, relativas às características e condições dos pacientes, foram: idade, sexo, escolaridade, classificação econômica segundo os critérios da Associação Brasileira de Empresas de Pesquisa (ABEP; http://www. abep.org.br, acessado em 22/Set/2006) e classificação da doença de acordo com a gravidade (intermitente, persistente leve, moderada e grave), conforme os critérios recomendados pelas IV Diretrizes Brasileiras de Manejo da Asma da Sociedade Brasileira de Pneumologia ${ }^{14}$. Todas as 
análises foram feitas comparando as USF com e sem programa considerado consolidado.

Os dados coletados foram digitados duplamente em bancos de dados no programa Epi Info, versão 3.2.2 (Centers for Disease Control and Prevention, Atlanta, Estados Unidos), para comparação e correções. As associações entre as variáveis foram analisadas pelo teste de qui-quadrado, com correção (Yates) quando possível, ou com o teste exato de Fischer nos casos em que ocorreram freqüências esperadas menores que 5. Em todos os testes foi adotado o nível de significância de $5 \%$. Também foi utilizado o teste de Mantel-Haenszel para controlar uma possível influência da idade no indicador de resultado atendimentos de urgência por asma e no indicador de processo uso de broncodilatador.

O estudo foi aprovado pelo Comitê de Ética em Pesquisa da Universidade Estadual de Londrina e cumpriu as normas da Resolução no. 196/96 do Conselho Nacional de Saúde. Foi solicitada, aos entrevistados ou seus responsáveis, a assinatura do termo de consentimento livre e esclarecido, após explicação dos objetivos da pesquisa.

\section{Resultados}

Da amostra selecionada na USF considerada com programa consolidado (212), 31 foram excluídos $(14,6 \%)$ - um por óbito e 30 por terem se mudado para outra área de abrangência. Dos 181 restantes, ocorreram 13 perdas $(7,2 \%)$, sendo duas por recusa em participar da entrevista e o restante por ausência em até três visitas ou por endereço não localizado. Assim, 168 pessoas foram entrevistadas. Na USF com programa considerado não consolidado (amostra de 165), houve quatro exclusões por mudanças de residências para outras áreas de abrangência e, dos 161 restantes, perda de 16 casos $(9,9 \%)$, sendo uma recusa e o restante por não estar presente no domicílio em até três visitas ou por mudança para área ignorada, totalizando 145 entrevistas ao final do estudo.

Observa-se, na Tabela 1, que não houve diferença significativa entre as USF em relação ao sexo dos inscritos. Entre os cadastrados nas USF com programa consolidado e não consolidado, mais da metade era composta pelo sexo feminino: $60,1 \%$ e $53,8 \%$, respectivamente. No entanto, diferença significativa ( $p<0,05)$ foi observada para a faixa etária. Na USF com programa consolidado, ocorreu predominância da faixa etária entre 15 e 59 anos $(51,2 \%)$, com menor proporção da faixa etária entre 2 e 6 anos (12,5\%). Comparativamente, nas USF com programa não consolidado também existiu predomínio da faixa etária de 15 a 59 anos (38,6\%); porém, a pro- porção de crianças com idades entre 2 e 6 anos foi quase o dobro $(21,4 \%)$ à da USF com programa consolidado.

A maioria dos entrevistados tinha menos de nove anos de estudo e classificava-se na classe econômica $\mathrm{C}$, sem diferenças entre os dois tipos de USF. Também não houve diferença significativa quanto à classificação da gravidade da asma, com predominância da asma persistente leve e moderada em ambos os tipos de USF, havendo, contudo, maior freqüência de asma intermitente nas USF com programa não consolidado $(15,9 \%)$ em comparação à USF com programa consolidado $(7,7 \%)$.

Os indicadores de processo são mostrados na Tabela 2. A proporção de pacientes cadastrados na USF com programa consolidado que referiu participar de reuniões de grupos para asmáticos foi de $32,1 \%$, significativamente maior ( $\mathrm{p}<$ $0,001)$ que nas USF com programa não consolidado (2\%). A diferença entre os tipos de USF foi altamente significativa quanto ao relato de recebimento de visita domiciliar, pois $81(48,2 \%)$ dos pacientes da USF com programa consolidado relataram ter recebido pelo menos uma visita domiciliar de algum membro da equipe da USF para orientação de controle ambiental, enquanto nenhum dos cadastrados nas USF com programa não consolidado referiu ter recebido tal visita. Cerca de $35 \%$ dos asmáticos cadastrados nas USF com e sem programa consolidado relataram atendimento fisioterápico.

O uso de corticóide inalatório foi semelhante entre os cadastrados em ambos os tipos de USF, entretanto o de broncodilatador foi maior $(\mathrm{p}<$ $0,001)$ nas USF com programa não consolidado (74,5\%), em comparação aos da USF com programa consolidado $(55,4 \%)$.

Quanto aos indicadores de resultado/impacto, observou-se que a proporção de alta foi maior (23,8\%) e a proporção de abandono foi menor (20,8\%) na USF com programa consolidado, em comparação às USF com programa não consolidado (alta de $8,2 \%$ e abandono de $36,6 \%$ ). A inscrição de portadores de asma na USF com programa considerado consolidado mostrou um efeito protetor para o abandono, com razão de prevalência $(\mathrm{RP})$ de $0,57(\mathrm{p}=0,003)$, conforme mostra a Tabela 3.

Não foram evidenciadas diferenças significativas entre as USF para relato de ocorrência de internação hospitalar por asma ou por outras infecções respiratórias (Tabela 3). Já em relação aos atendimentos por asma em serviços de urgência, verifica-se que houve diferença estatisticamente significativa. Dos pacientes asmáticos inscritos nas USF com programa não consolidado, 81 (55,9\%) referiram atendimentos de urgência 
Distribuição dos asmáticos cadastrados nas unidades de saúde da família (USF) com programa consolidado e não consolidado segundo características sócio-demográficas e quanto à classificação de gravidade da asma. Londrina, Paraná, Brasil, 2007.

\begin{tabular}{|c|c|c|c|c|c|}
\hline \multirow[t]{3}{*}{ Características } & \multicolumn{4}{|c|}{ Tipo de USF (programa) } & \multirow[t]{3}{*}{ Valor de $p$ * } \\
\hline & \multicolumn{2}{|c|}{ Consolidado } & \multicolumn{2}{|c|}{ Não consolidado } & \\
\hline & $\mathbf{n}$ & $\%$ & $\mathbf{n}$ & $\%$ & \\
\hline \multicolumn{6}{|l|}{ Sexo } \\
\hline Masculino & 67 & 39,9 & 67 & 46,2 & 0,310 \\
\hline Feminino & 101 & 60,1 & 78 & 53,8 & \\
\hline \multicolumn{6}{|l|}{ Faixa etária (anos) } \\
\hline $2-6$ & 21 & 12,5 & 31 & 21,4 & 0,041 \\
\hline $7-14$ & 32 & 19,0 & 37 & 25,5 & \\
\hline $15-59$ & 86 & 51,2 & 56 & 38,6 & \\
\hline 60 e mais & 29 & 17,3 & 21 & 14,5 & \\
\hline \multicolumn{6}{|c|}{ Escolaridade (anos de estudo) ** } \\
\hline$<9$ & 123 & 73,2 & 92 & 63,4 & 0,082 \\
\hline 9 ou mais & 45 & 26,8 & 53 & 36,6 & \\
\hline \multicolumn{6}{|l|}{ 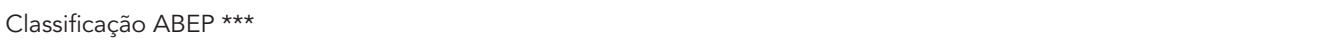 } \\
\hline$A-B$ & 32 & 19,1 & 17 & 11,7 & 0,194 \\
\hline $\mathrm{C}$ & 95 & 56,5 & 87 & 60,0 & \\
\hline D-E & 41 & 24,4 & 41 & 28,3 & \\
\hline \multicolumn{6}{|c|}{ Classificação de gravidade da asma } \\
\hline Intermitente & 13 & 7,7 & 23 & 15,9 & 0,149 \\
\hline Persistente leve & 87 & 51,8 & 71 & 49,0 & \\
\hline Persistente moderada & 47 & 28,0 & 37 & 25,5 & \\
\hline Persistente grave & 21 & 12,5 & 14 & 9,6 & \\
\hline
\end{tabular}

* Teste de qui-quadrado, com correção de Yates quando aplicável;

** Em menores de 12 anos foi considerada escolaridade da mãe ou responsável;

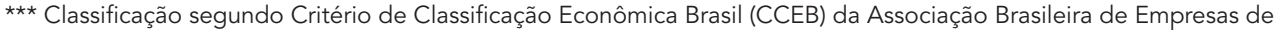

Pesquisa (ABEP; http://www.abep.org.br, acessado em 22/Set/2006).

devidos à exacerbação da asma, enquanto na USF com programa consolidado foram 49 (29,2\%).

A Tabela 4 apresenta a prevalência de atendimentos de urgência por asma por faixa etária. Verifica-se que a prevalência global ajustada pela faixa etária de atendimentos de urgência na USF com programa consolidado é inferior à das USF com programa não consolidado, com RP ajustada pelo teste de Mantel-Haenszel de 0,54, e intervalo de 95\% de confiança (IC95\%) de 0,41-0,71. Destaca-se, todavia, maior efeito do programa na faixa etária dos 15 a 59 anos.

Pode-se observar (Tabela 5) que, mesmo controlando a faixa etária, houve associação entre o uso de broncodilatador e o tipo de USF (RP ajustada = 0,74, IC95\%: 0,63-0,87; p < 0,001). De forma semelhante à prevalência de atendimentos de urgência, o efeito foi mais visível na faixa etária dos 15 a 59 anos.

\section{Discussão}

Os resultados encontrados no presente estudo evidenciaram algumas diferenças significativas entre as USF com programa consolidado e não consolidado, confirmando o pressuposto de que um programa de controle da asma precisa ser efetivo para ter maior impacto nos indicadores. No entanto, tais diferenças poderiam ser ainda maiores se fossem comparadas USF com e sem programa de asma, pois também ficou evidenciado que mesmo as USF com programa não consolidado realizavam algumas ações, e estas podem ter influenciado nos achados, resultando em um número menor de crises.

A USF considerada como com programa consolidado apresentou melhor organização das ações, como esperado, pois apresentou maior participação dos pacientes em grupos de asmáticos e realização de visitas domiciliares para 
Distribuição dos asmáticos segundo variáveis relacionadas às atividades do programa de controle da asma e uso de medicamentos em unidades de saúde da família (USF) com programa consolidado e não consolidado. Londrina, Paraná, Brasil, 2007.

\begin{tabular}{|c|c|c|c|c|c|}
\hline \multirow[t]{3}{*}{ Características } & \multicolumn{4}{|c|}{ Tipo de USF (programa) } & \multirow[t]{3}{*}{ Valor de $p$ * } \\
\hline & \multicolumn{2}{|c|}{ Consolidado } & \multicolumn{2}{|c|}{ Não consolidado } & \\
\hline & n & $\%$ & $\mathbf{n}$ & $\%$ & \\
\hline \multicolumn{6}{|c|}{ Participação em grupos de asmáticos } \\
\hline Sim & 54 & 32,1 & 3 & 2,1 & $<0,001 *$ \\
\hline Não & 114 & 67,9 & 142 & 97,9 & \\
\hline \multicolumn{6}{|l|}{ Visita domiciliar } \\
\hline Sim & 81 & 48,2 & - & - & $<0,001 * \star$ \\
\hline Não & 87 & 51,8 & 145 & 100,0 & \\
\hline \multicolumn{6}{|c|}{ Atendimento fisioterápico } \\
\hline Sim & 62 & 36,9 & 51 & 35,2 & 0,841 * \\
\hline Não & 106 & 63,1 & 94 & 64,8 & \\
\hline \multicolumn{6}{|c|}{ Corticóide inalatório *** } \\
\hline Sim & 73 & 43,5 & 67 & 46,2 & 0,707 * \\
\hline Não & 95 & 56,5 & 78 & 53,8 & \\
\hline \multicolumn{6}{|c|}{ Uso de broncodilatador \# } \\
\hline Sim & 93 & 55,4 & 108 & 74,5 & $<0,001$ * \\
\hline Não & 75 & 44,6 & 37 & 25,5 & \\
\hline
\end{tabular}

* Teste de qui-quadrado com correção de Yates;

** Teste exato de Fischer;

*** Nos últimos 30 dias;

\# Nos últimos 3 meses.

Prevalências, respectivas razões de prevalência (RP) e intervalo de $95 \%$ de confiança (IC95\%) relacionados aos indicadores de resultado/impacto entre pacientes cadastrados em unidades de saúde da família (USF) com programa consolidado e não consolidado. Londrina, Paraná, Brasil, 2007.

\begin{tabular}{|c|c|c|c|c|c|c|c|}
\hline \multirow[t]{3}{*}{ Variável } & \multicolumn{4}{|c|}{ Tipo de USF (programa) } & \multirow[t]{3}{*}{ RP } & \multirow[t]{3}{*}{ IC95\% } & \multirow[t]{3}{*}{ Valor de p * } \\
\hline & \multicolumn{2}{|c|}{ Consolidado } & \multicolumn{2}{|c|}{ Não consolidado } & & & \\
\hline & n & $\%$ & $\mathbf{n}$ & $\%$ & & & \\
\hline Abandono do programa & 35 & 20,8 & 53 & 36,6 & 0,57 & $0,40-0,82$ & 0,003 \\
\hline Internação por asma ** & 22 & 13,1 & 14 & 9,7 & 1,35 & $0,72-2,55$ & 0,439 \\
\hline Internação por IRA ** & 13 & 7,7 & 14 & 9,7 & 0,80 & $0,38-1,64$ & 0,688 \\
\hline 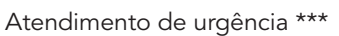 & 49 & 29,2 & 81 & 55,9 & 0,52 & $0,40-0,69$ & $<0,001$ \\
\hline
\end{tabular}

IRA: infecção respiratória aguda.

* Teste de qui-quadrado com correção de Yates;

** Nos últimos 12 meses:

*** Nos últimos 3 meses.

orientação de controle ambiental. Além disso, notou-se também menor uso de broncodilatador, empregado para reverter situações de crises, o que possivelmente reflete melhor organização das ações e maior adesão dos pacientes às re- comendações preconizadas pelo programa para prevenção de crises. Conseqüentemente, aconteceram menores proporções de abandono do programa, de relatos de crises asmáticas e de visitas aos serviços de urgência. Esses resultados 
Prevalência de atendimentos de urgência devidos à asma segundo faixa etária dos asmáticos cadastrados em unidades de saúde da família (USF) com programa consolidado e não consolidado e respectivas razões de prevalência (RP) e intervalo de 95\% de confiança (IC95\%). Londrina, Paraná, Brasil, 2007.

\begin{tabular}{|c|c|c|c|c|c|c|c|c|c|}
\hline \multirow[t]{3}{*}{ Variável } & \multicolumn{6}{|c|}{ Tipo de USF (programa) } & \multirow[t]{3}{*}{$\mathrm{RP}$} & \multirow[t]{3}{*}{ IC95\% } & \multirow[t]{3}{*}{ Valor de $p$ * } \\
\hline & \multicolumn{3}{|c|}{ Consolidado } & \multicolumn{3}{|c|}{ Não consolidado } & & & \\
\hline & $\mathrm{T}$ & $\mathbf{n}$ & $\%$ & $\mathrm{~T}$ & $n$ & $\%$ & & & \\
\hline $2-6$ & 21 & 10 & 47,6 & 31 & 18 & 58,1 & 0,86 & $0,51-1,46$ & 0,782 \\
\hline $7-14$ & 32 & 9 & 28,1 & 37 & 19 & 51,4 & 0,55 & $0,29-1,04$ & 0,087 \\
\hline $15-59$ & 86 & 22 & 25,6 & 56 & 34 & 60,7 & 0,42 & $0,28-0,64$ & $<0,001$ \\
\hline 60 e mais & 29 & 8 & 27,6 & 21 & 10 & 47,6 & 0,58 & $0,28-1,22$ & 0,247 \\
\hline Total * & 168 & 49 & 29,2 & 145 & 81 & 55,9 & 0,54 & $0,41-0,71$ & $<0,001$ \\
\hline
\end{tabular}

Nota: qui-quadrado para heterogeneidade $(p=0,231)$.

* Estimativas ajustadas pelo teste de Mantel-Haenszel.

\section{Tabela 5}

Prevalência de uso de broncodilatador segundo faixa etária dos asmáticos cadastrados em unidades de saúde da família (USF) com programa consolidado e não consolidado e respectivas razões de prevalência (RP) e intervalo de 95\% de confiança (IC95\%). Londrina, Paraná, Brasil, 2007.

\begin{tabular}{|c|c|c|c|c|c|c|c|c|c|}
\hline \multirow[t]{3}{*}{ Variável } & \multicolumn{6}{|c|}{ Tipo de USF (programa) } & \multirow[t]{3}{*}{ RP } & \multirow[t]{3}{*}{ IC95\% } & \multirow[t]{3}{*}{ Valor de $p$ * } \\
\hline & \multicolumn{3}{|c|}{ Consolidado } & \multicolumn{3}{|c|}{ Não consolidado } & & & \\
\hline & $\mathrm{T}$ & $\mathbf{n}$ & $\%$ & $\mathrm{~T}$ & $\mathbf{n}$ & $\%$ & & & \\
\hline $2-6$ & 21 & 15 & 71,4 & 31 & 22 & 71,0 & 1,01 & $0,71-1,43$ & 0,783 \\
\hline $7-14$ & 32 & 15 & 46,9 & 37 & 22 & 59,5 & 0,79 & $0,50-1,24$ & 0,422 \\
\hline $15-59$ & 86 & 45 & 52,3 & 56 & 46 & 82,1 & 0,64 & $0,50-0,81$ & $<0,001$ \\
\hline 60 e mais & 29 & 18 & 62,1 & 21 & 18 & 85,7 & 0,72 & $0,52-1,01$ & 0,129 \\
\hline Total * & 168 & 93 & 55,4 & 145 & 108 & 74,5 & 0,74 & $0,63-0,87$ & $<0,001$ \\
\hline
\end{tabular}

Nota: qui-quadrado para heterogeneidade $(p=0,206)$.

* Estimativas ajustadas pelo teste de Mantel-Haenszel.

indicam que não basta apenas ter programas implantados; as equipes devem empenhar-se para consolidar seus programas, de modo que eles funcionem de acordo com as recomendações dos protocolos.

Cabe ressaltar que as USF selecionadas para este estudo receberam o mesmo modelo de treinamento teórico-prático, realizado pelos mesmos profissionais, mas são diferentes em seus processos de trabalho. Portanto, há necessidade de outros estudos, preferencialmente incorporando aspectos subjetivos da avaliação em saúde, com participação dos atores sociais envolvidos, especialmente dos usuários dos serviços 14, que possam explicar melhor essas diferenças entre as USF.

No presente estudo, para $36,5 \%$ e $54,4 \%$ das crianças de 2 a 14 anos das USF com e sem programa consolidado, respectivamente, houve re- lato de necessidade de atendimento de urgência. Ainda que a USF com programa consolidado também apresentasse deficiências, encontrou-se maior impacto nas agudizações da asma. Um estudo de coorte realizado na Bahia também mostrou que, após implantação de um programa de controle da asma grave, houve redução de $85 \%$ dos atendimentos de urgência e de $90 \%$ no número de internações hospitalares 15.

Os resultados do presente trabalho ainda permitem aventar a hipótese de que mesmo um programa não consolidado pode reduzir a necessidade de atendimentos de urgência. Observou-se, no presente estudo, que os percentuais de atendimento de urgência encontrados nas duas USF estudadas sem programa consolidado foram ainda menores do que os observados por Camargos \& Profeta 16 em Belo Horizonte (Minas Gerais), onde $92,7 \%$ das crianças de 4 a 14 anos haviam 
procurado atendimento em serviços de urgência em um período de doze meses anteriores à admissão em ambulatório de pneumologia.

Estudo realizado por Sarinho et al. 17, em Recife (Pernambuco), verificou que a maioria dos pacientes asmáticos ainda era atendida exclusivamente em serviços de urgência, evidenciando que, apesar da importância do acompanhamento preventivo, ele se encontra longe de ser uma realidade acessível a toda população. $\mathrm{O}$ sofrimento e os gastos com internações e atendimentos de urgência poderiam ser minimizados se o acesso aos serviços de saúde em nível ambulatorial fosse facilitado, e se fosse efetivamente implantada uma política nacional de acompanhamento dos pacientes com asma. Todavia, os serviços de urgência ainda são utilizados como principal local de atendimento pelos asmáticos em nosso país 18,19 .

Em Porto Alegre (Rio Grande do Sul), um estudo 20 comparou a freqüência de exacerbação da asma em pacientes atendidos em serviços de urgência com outros atendidos em ambulatórios. Verificou-se uma proporção de 95,3\% de visitas aos departamentos de urgência em um período de doze meses anteriores à realização da pesquisa entre aqueles que freqüentavam apenas os serviços de urgência, em comparação a 48,8\% entre acompanhados ambulatorialmente.

O uso de corticóide inalatório por pacientes asmáticos foi semelhante nas USF selecionadas: quase metade dos asmáticos cadastrados em cada tipo de USF estava utilizando o medicamento. No entanto, estudo realizado em Porto Alegre 21 mostrou que $98,5 \%$ dos asmáticos pesquisados não realizavam tratamento com corticóide intercrise, e que $1,5 \%$ dos que faziam tratamento medicamentoso recebia drogas não preconizadas pelos consensos de manejo da asma.

O tratamento profilático com uso de corticóides inalatórios deve ser avaliado por parâmetros clínicos, como intensidade dos sintomas, capacidade de exercer atividades diárias e a necessidade de uso de broncodilatador 22. Lasmar et al. 23 estudaram crianças e adolescentes com asma e verificaram que $94,2 \%$ não utilizavam nenhum tipo de medicação profilática. O tratamento da asma, além de englobar ações educativas, deve ser realizado com medicamentos profiláticos intercrise. Os broncodilatadores devem ser utilizados no tratamento da asma com o objetivo de reverter a constrição que ocorre nos momentos de crise, preferencialmente por via inalatória 24 .

Neste estudo, não houve diferença quanto ao relato de internação hospitalar entre portadores de asma cadastrados nas USF com programa consolidado $(13,1 \%)$ e não consolidado $(9,7 \%)$. Entretanto, proporção maior de asmáticos ins- critos na USF com programa consolidado relatou internações hospitalares por asma. Este fato talvez se explique por esta USF ter apresentado proporção um pouco maior de asmáticos graves, fato que pode ter elevado a proporção de internação. Além disso, mesmo tendo sido considerada USF com programa consolidado, houve uma taxa considerável de abandonos (20\%), que pode ter influenciado na taxa de internação. Porém, os resultados foram inferiores aos encontrados por outros autores. Chatkin et al. 18, analisando os fatores de risco para consultas em pronto-socorro em Pelotas (Rio Grande do Sul), mostraram que $26 \%$ das crianças estudadas haviam sido internadas no último ano. Em Minas Gerais, uma pesquisa 15 analisando 560 crianças asmáticas em um ambulatório, constatou que $42,7 \%$ dessas crianças haviam sido internadas nos últimos 12 meses.

Quanto à classificação da gravidade, a grande maioria dos asmáticos cadastrados nos dois tipos de USF foi classificada como portador de asma do tipo intermitente e persistente leve, seguidos de moderados e graves. Não houve diferença, neste estudo, entre as USF em relação à gravidade da doença. Estudo realizado em Embu (São Paulo) 25, com crianças e adolescentes de 0 a 14 anos, também encontrou predominância de asma leve, e os classificados como graves apresentaram uma freqüência em torno de $10 \%$, resultado semelhante ao observado em nosso estudo. Os resultados do nosso estudo foram semelhantes ao que se espera encontrar na população asmática em geral. Estima-se que $60 \%$ dos casos de asma sejam intermitentes ou persistentes leves, $25 \%$ a $30 \%$ moderados e $5 \%$ a $10 \%$ graves 4 . Este resultado mostra que maiores esforços devem ser feitos para o tratamento do portador de asma persistente moderada nas USF, visto que, segundo as diretrizes e protocolos do Ministério da Saúde, este nível de gravidade da doença deve ser manejado na atenção primária.

Ainda em relação aos atendimentos nos serviços de urgência, a freqüência foi maior na faixa etária de 2 a 6 anos para a USF com programa consolidado, fato este também encontrado em outros estudos 23,26. Na USF com programa não consolidado, a freqüência de atendimentos de urgência foi ainda maior que na USF com programa consolidado para todas as faixas etárias. A análise dos resultados nos mostra que o fato de o paciente com asma ser cadastrado na USF com programa consolidado foi fator de proteção para atendimentos de urgência.

Os resultados deste estudo mostraram que as populações das USF são comparáveis, e que esse perfil não justifica as diferenças encontradas nas USF com programa consolidado e não 
consolidado. As regiões são semelhantes quanto à situação sócio-econômica, conforme a classificação econômica da ABEP, e também quanto ao grau de escolaridade. Mesmo controlando diferenças relativas à faixa etária, verificou-se maior ocorrência de atendimentos de urgência nas USF com programa não consolidado.

Uma das limitações deste estudo pode ter sido o viés de memória, embora este fator tenha sido minimizado com a determinação de tempo para ocorrência dos eventos de 12 meses para as internações hospitalares e de três meses anteriores à realização da pesquisa para os atendimentos de urgência e uso de broncodilatadores. Tais fatores também foram amenizados por se tratar de eventos marcantes, o que facilita a recordação. Outra limitação do estudo pode ter sido o tamanho da amostra, que não foi calculada para demonstrar eventos de baixa freqüência, como por exemplo, as internações hospitalares.

Há ainda que se considerar que a coleta de dados, no presente estudo, ocorreu predominantemente no verão (fevereiro e março), seguido do outono (março até início de maio) e que diferenças nas condições climáticas podem ter tido impacto nos desfechos analisados. O município estudado, localizado a latitude e longitude aproximadas de $23^{\circ} \mathrm{S}$ e $51^{\circ} \mathrm{O}$, respectivamente, apresenta clima subtropical úmido mesotérmico, com temperaturas médias mensais mais semelhantes às apresentadas na Região Sudeste, especialmente no Estado de São Paulo e no sul de Minas Gerais, do que às da maioria dos estados da Região Sul, incluindo outras cidades mais ao sul do Estado do Paraná. Estudo realizado em São Paulo observou maior número de internações por asma nos meses de outono (entre mar- ço e maio) 27 e outro, conduzido em Juiz de Fora (Minas Gerais), analisando 25.243 atendimentos de crise asmática em serviços públicos ambulatoriais e de urgência/emergência, constatou maiores números de atendimentos nos meses de maio, junho e julho 28 . Todavia, há de se destacar que, na presente investigação, a coleta de dados foi feita pela equipe de pesquisadores de forma simultânea em todas as USF, o que possivelmente controlou o efeito das alterações climáticas na análise das diferenças entre as USF.

Embora ainda sejam poucas as experiências com programas de controle da asma em nosso país, este estudo mostra que a implantação do programa "Respira Londrina" foi uma intervenção que resultou em melhoria das condições de vida dos pacientes com asma, refletindo também melhores indicadores de saúde para o município. No entanto, o presente trabalho também indica que, para ser mais efetivo em termos de redução de atendimentos de urgência e de uso de broncodilatadores, o programa precisa estar bem estruturado em cada USF do município.

$\mathrm{Na}$ perspectiva de minimizar os problemas da asma, bastante ênfase tem se dado aos programas de controle dessa doença, os quais têm comprovado ser capazes de reduzir hospitalizações e atendimentos em urgências 6,15, melhorando a qualidade de vida das pessoas que convivem com essa doença. A asma é uma das condições mais prevalentes sensíveis às ações de atenção primária à saúde em nosso meio 29 , com intuito de reduzir internações evitáveis. Assim, estratégias semelhantes devem ser estimuladas para que se amplie o acesso de portadores de asma brônquica a esse tipo de programa em outras localidades.

\section{Resumo}

O objetivo deste estudo foi avaliar o programa de controle da asma (programa "Respira Londrina") do Municipio de Londrina, Paraná, Brasil. Trata-se de estudo transversal, comparando unidade de saúde da família (USF) com o programa consolidado e duas USF sem o programa consolidado. Foram entrevistados 313 asmáticos, 168 da USF com programa consolidado e 145 das USF com programa não consolidado. Na amostra estudada, houve diferenças significativas $(p<0,001)$ na utilização de broncodilatadores e no número de atendimentos de urgência nas USF com programa consolidado e não consolidado. Dos asmáticos inscritos na USF com programa consolidado, 55,4\% referi- ram uso de broncodilatador em comparação a 74,5\% dos inscritos nas USF com programa não consolidado. Respectivamente, 29,2\% e 55,9\% dos pacientes das USF com programa consolidado e não consolidado necessitaram de atendimento de urgência. Um programa de controle da asma bem estruturado pode resultar na redução dos atendimentos de urgência decorrentes de crises asmáticas, contribuindo na melhoria dos indicadores de saúde, bem como para a elevação da qualidade de vida.

Asma; Avaliação de Programas e Projetos de Saúde; Serviços Médicos de Emergência; Hospitalização 


\section{Colaboradores}

T. A. Carmo foi responsável pela coleta de dados e pela redação da versão preliminar do artigo. Também foi responsável pelo delineamento da pesquisa, análise de dados, revisão crítica e aprovação da versão final do artigo. S. M. Andrade e A. Cerci Neto participaram do delineamento da pesquisa, análise de dados, revisão crítica e aprovação da versão final do artigo.

\section{Agradecimentos}

Ao professor Darli Antonio Soares (in memoriam) pela orientação segura e competente dada durante o desenvolvimento deste trabalho.

\section{Referências}

1. Mallol J, Solé D, Ascher I, Clayton T, Stein R, SotoQuiroz M. Prevalence of asthma symptoms in Latin America: the International Study of Asthma and Allergies in Childhood (ISAAC). Pediatr Pulmonol 2000; 30:439-44.

2. Solé D, Yamada E, Vana AT, Werneck G, Solano de Freitas L, Sologuren MJ, et al. International study of asthma and allergies in childhood (ISAAC): Prevalence of asthma and asthma-related symptoms among Brazilian schoolchildren. J Investig Allergol Clin Immunol 2001; 11:123-8.

3. Castro LKK. Prevalência de asma, de rinite e de eczema atópico em escolares da zona urbana de Londrina - PR [Dissertação de Mestrado]. Londrina: Programa de Pós-graduação em Medicina e Ciências da Saúde, Universidade Estadual de Londrina; 2009.

4. Sociedade Brasileira de Pneumologia e Tisiologia. III consenso brasileiro no manejo da asma. J Pneumol 2002; 28:S1-28.
5. Macedo SEC, Menezes AMB, Knorst M, Dias-daCosta JS, Gigante DP, Olinto MTA, et al. Fatores de risco para a asma em adultos, Pelotas, Rio Grande do Sul, Brasil. Cad Saúde Pública 2007; 23:863-74.

6. Cerci Neto A. Mudança no perfil do manejo da asma em uma cidade brasileira de médio porte após programa estruturado: dados após quatro anos de implantação [Tese de Doutorado]. Londrina: Programa de Pós-graduação em Medicina e Ciências da Saúde, Universidade Estadual de Londrina; 2009.

7. Global Initiative for Asthma. Global strategy for asthma management and prevention; 2009. http:// www.ginasthma.com/Guidelineitem.asp??11= 2\&l2=1\&intId=1561 (acessado em 17/Mai/2010).

8. Oliveira MA, Muniz MT, Santos LA, Faresin SM, Fernandes ALG. Custo-efetividade de programa de educação para adultos asmáticos atendidos em hospital-escola de instituição pública. J Pneumol 2002; 28:71-6. 
9. Méndez NHS, Moralez DTJ, Campos JJM, Ortega JM, Vázquez JUO, Delgado V, et al. Resultados de um programa educativo para adultos asmáticos. Rev Alerg Mex 2001; 48:42-4.

10. Cerci Neto A, organizador. Asma em saúde pública. São Paulo: Editora Manole; 2007.

11. Gerência de Informações em Saúde, Autarquia Municipal de Saúde. Estimativas preliminares dos totais populacionais por UBS. Londrina: Autarquia Municipal de Saúde; 2006.

12. Donabedian A. The quality of care. How can it be assessed? JAMA 1988; 260:1743-8.

13. IV diretrizes brasileiras para o manejo da asma. J Bras Pneumol 2006; 32 Suppl 7:S447-74.

14. Uchimura KY, Bosi MLM. Qualidade e subjetividade na avaliação de programas e serviços em saúde. Cad Saúde Publica 2002; 18:1561-9.

15. Ponte E, Franco RA, Souza-Machado A, Souza-Machado C, Cruz AA. Impacto de um programa para o controle da asma grave na utilização de recursos do Sistema Único de Saúde. J Bras Pneumol 2007; 33:15-9.

16. Camargos PAM, Profeta SC. Tratamento de manutenção da asma persistente à admissão em ambulatório de pneumologia pediátrica. J Pediatr (Rio J.) 2003; 79:233-8.

17. Sarinho E, Queiroz GRS, Dias MLCM, Silva AJQ. A hospitalização por asma e a carência de acompanhamento ambulatorial. J Bras Pneumol 2007; 33:365-71.

18. Chatkin M, Menezes AMB, Albernaz E, Victora CG, Barros FC. Fatores de risco para consultas em pronto-socorro por crianças asmáticas no Sul do Brasil. Rev Saúde Pública 2000; 34:491-8.

19. Lasmar LMLBF, Fontes MJF, Guerra HL, Jentzsch NS. Perfil da assistência pública à criança e ao adolescente asmáticos. Rev Méd Minas Gerais 2000; 10:219-31.
20. Dalcin PTR, Piovesan DM, Kang S, Fernandes AK, Franciscatto E, Millan T, et al. Factors associated with emergency department visits due to acute asthma. Braz J Med Biol Res 2004; 37:1331-8.

21. Ache BICS, Kahan F, Fiterman J. Prevalência de sintomas de asma e tratamento de crianças e adolescentes de 2 a 14 anos no Campus Aproximado da PUCRS. J Bras Pneumol 2005; 31:103-10.

22. Moura JAR, Camargos PAM, Blic J. Tratamento profilático da asma. J Pediatr (Rio J.) 2002; 78:141-50.

23. Lasmar L, Goulart E, Sakurai E, Camargos P. Fatores de risco para hospitalização de crianças e adolescentes asmáticos. Rev Saúde Pública 2002; 36:409-19.

24. Rosário Filho NA. Broncodilatadores na asma: contornando controvérsias. J Pediatr (Rio J.) 1996; 72:281-6.

25. Ventura RN, Naspitz C, Puccini RF, Silva EMK. Avaliação do programa de atenção a crianças asmáticas acompanhadas nas unidades de saúde do $\mathrm{Mu}$ nicípio do Embu, São Paulo, no período de 1988 a 1993. Cad Saúde Pública 1998; 14:117-28.

26. Stein RT. Asma pediátrica - o impacto das internações hospitalares. J Bras Pneumol 2006; 32:25-6.

27. Toyoshima MTK, Ito GM, Gouveia N. Morbidade por doenças respiratórias em pacientes hospitalizados em São Paulo/SP. Rev Assoc Méd Bras 2005; 51:209-13.

28. Ezequiel OS, Gazeta GS, Freire NMS. Prevalência dos atendimentos por crises de asma nos serviços públicos do Município de Juiz de Fora (MG). J Bras Pneumol 2007; 33:20-7.

29. Alfradique ME, Bonolo PF, Dourado I, Lima-Costa MF, Macinko J, Mendonça CS, et al. Internações por condições sensíveis à atenção primária: a construção da lista brasileira como ferramenta para medir o desempenho do sistema de saúde (Projeto ICSAP - Brasil). Cad Saúde Pública 2009; 25:1337-49.

Recebido em 07/Jun/2010

Versão final reapresentada em 16/Set/2010

Aprovado em 28/Set/2010 